\title{
Spectrum-Sensing Opportunistic Wireless Relay Networks: Outage and Diversity Performance
}

\author{
Kyounghwan Lee Aylin Yener \\ Wireless Communications and Networking Laboratory \\ Electrical Engineering Department \\ The Pennsylvania State University \\ University Park, PA 16802 \\ kxl251@psu.eduyener@ee.psu.edu
}

\begin{abstract}
We consider a spectrum-sensing opportunistic wireless relay network which is defined by a source node, a destination node, and a group of network clusters each of which consists of a number of cognitive (unlicensed) relay nodes and a primary (licensed) node. In this network, the cognitive nodes can help the source node via a number of possible relaying techniques in an opportunistic fashion, i.e., by acquiring unused spectrum nominally assigned to a primary node. We aim to understand the performance of this system by investigating the impact of spectrum acquisition on the diversity order provided by the cognitive relay nodes. We consider three relay transmission schemes, namely, regenerative decode-and-forward, non-regenerative decode-andforward and amplify-and-forward under fairly general channel conditions. We find that, regardless of the relay transmission scheme used, the imperfections that may arise during acquisition of the spectrum by the cognitive nodes can cause a significant reduction in diversity order. The good news is that this penalty can be compensated for if a sufficient number of potential relay nodes within a cluster cooperatively sense the spectrum and help the designated cognitive relay node.
\end{abstract}

\section{INTRODUCTION}

The demand for radio spectrum is continuing to grow with new emerging applications and further market penetration. Currently, the radio spectrum is regulated and the licensed spectrum bands are not "shared". On the other hand, the licensed resources may not be in use continuously, leading to possible under-utilization of the spectrum that is in high demand [1]. In a more flexible scenario, one can envision that the licensed resources, whenever not in use, can be captured and used by additional users, increasing the efficiency of spectrum use. To this end, cognitive radios which are amenable to employ a more open spectrum policy have attracted considerable attention recently [2], [3].

A cognitive radio allows a cognitive (unlicensed) user to access a spectrum hole unoccupied by a primary (licensed) user and improve the spectrum utilization while reducing the white spaces in the spectrum [3]. A requirement for this system is seamless operation; thus, the cognitive users must detect the presence of the spectrum hole, i.e., equivalently, detect the presence of the primary user's transmission [3], [4]. The common approach for detecting these unknown signals is to use an energy detector [5], [6]. Recent research effort investigates the scenario where a group of neighboring nodes cooperate with a desired cognitive user to improve the spectrum sensing performance. It has been shown that such cooperative spectrum sensing provides reliable and fast detection of the presence of primary users [7], [8].

The notion of cognitive radios opportunistically capturing spectrum and communicating wirelessly, although mostly thought of in the context of cellular systems up to date, is equally intriguing for multihop wireless networks. As wireless networks are continuing to evolve towards allowing mobile nodes to communicate without the need for infrastructure while providing more reliability and increased capacity, relay networks, where a source node is assisted by intermediate nodes, offer a significant performance gain advantage [9]-[12]. Thus, inspired by the performance advantage of wireless relay networks, as well as the promise of opportunistic spectrum utilization, in this paper, we consider spectrum-sensing opportunistic wireless relay networks.

The spectrum sensing opportunistic relay network we consider consists of a source, a destination, and a group of network clusters each of which consists of a number of cognitive relay nodes and a primary node. The cognitive nodes relay information for the source node in an opportunistic fashion by acquiring spectrum unused by a primary node. Specifically, we aim to investigate the outage performance and understand the impact of spectrum acquisition performance of the cognitive relay nodes on the outage performance. Thus, we analyze the high SNR approximation of the outage performance in order to examine the diversity order of these networks. We should note here that in our recent work [13], we have studied the outage performance and diversity order for a simple symmetric cognitive relay network when the each cognitive relay node necessarily employs a particular relaying technique, namely, regenerative decode-and-forward. In this paper, we remove the assumption of symmetry in channel gains and consider a more general system model. Also, we consider a variety of relaying schemes, including non-regenerative decode-and-forward and amplify-and-forward.

Our conclusion is that regardless of the relaying technique used and assumptions on channel conditions, the following argument can be made: (i) the opportunistic scenario where relay nodes have to sense spectrum holes to help out the source node incurs a cooperative diversity order penalty as compared to a system where relay nodes have dedicated resources, and that (ii) this penalty can be compensated for if a sufficient number of potential relay nodes within the cluster 
cooperatively sense the spectrum and help the designated cognitive relay node.

\section{System ModeL}

We consider a wireless relay network where a source is assisted by a number of relay nodes. The relay nodes are cognitive (unlicensed) users and are grouped in $M$ clusters, based on their geographical proximity. Each cluster consists of a number of cognitive relay nodes (a potential relay node and neighboring relay nodes) and one primary (licensed) node. The system model is depicted in Figure 1. Among the nodes in a cluster, one is chosen as a potential relay node and can assist the source node, if the potential node is able to relay the message from the source, and acquire the spectrum hole unoccupied by its primary user successfully. We define the relaying set $R(s)$ to be the set of successful potential relays that meet both of these requirements. We consider three relaying techniques:

- Regenerative Decode-and-Forward (RDF): When the transmission from the source is received reliably at the relay node, the relay node decodes the signal, re-encodes it with the same codebook used in the source node and transmits the signal to the destination [12].

- Nonregenerative Decode-and-Forward (NDF): The relay node decodes the signal from the source reliably, but reencodes it using a different codebook than that of source [12].

- Amplify-and-Forward (AF): The relay node forwards a scaled version of the noisy copy of the source signal it received to the destination node [11].

Note that for a RDF or a NDF relay node to relay the source signal, it needs to be able to decode the signal reliably, whereas this is not necessary for AF. We assume that each primary user in a cluster communicates over an orthogonal frequency band with bandwidth $W$. The primary user broadcasts a beacon signal over a side channel to inform relay nodes of the availability of a frequency band. We note that even though the beacon requires an additional control channel and transmitted power, it is resource efficient for the relay nodes in that it prevents the potential nodes from browsing the entire frequency range. It also ensures that the cognitive nodes do not interfere with the primary nodes [14].

The communication takes place in two phases. In the first phase, the source broadcasts its message $\left(X_{s}\right)$ to each cluster and the destination node. In the second phase, the relay nodes relay to the destination node over the acquired spectrum. The received signals at each relay and the destination, respectively, during the first phase is given by

$$
Y_{r_{i}}=h_{s r_{i}} X_{s}+N_{s r_{i}}, \quad Y_{d}=h_{s d} X_{s}+N_{s d}
$$

During the second phase, the destination receives the signal from the successful potential relay node, in frequency band $i$

$$
Y_{d_{i}}=h_{r_{i} d} X_{r_{i}}+N_{r_{i} d}
$$

where $X_{r_{i}}$ is the transmitted signal from the successful potential relay node $i . N_{s r_{i}}, N_{s d}$, and $N_{r_{i} d}$ are zero-mean, independent, circularly symmetric, complex Gaussian random variables with variance $N_{0} . h_{s r_{i}}, h_{s d}$ and $h_{r_{i} d}$ are the fading

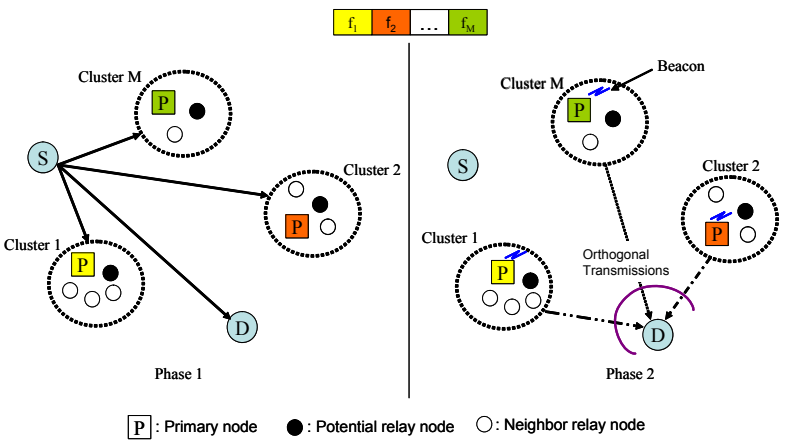

Fig. 1. System Model

coefficients and modeled as zero-mean, independent, circularly symmetric complex Gaussian random variables with variances $1 / \lambda_{s r_{i}}, 1 / \lambda_{s d}$, and $1 / \lambda_{r_{i} d}$, respectively. The source and relays all transmit with power $P$. We define the received SNR at the relay and the destination as:

$\gamma_{s r_{i}}=S N R\left|h_{s r_{i}}\right|^{2}, \gamma_{s d}=S N R\left|h_{s d}\right|^{2}, \gamma_{r_{i} d}=S N R\left|h_{r_{i} d}\right|^{2}$

where $S N R=P / N_{0} W$.

\section{Outage Probability AnAlysis}

For a slow fading channel, an appropriate metric is the outage capacity: one can talk about a tradeoff between the outage probability and the supportable rate. The outage occurs when the mutual information $(I)$ falls below a certain rate $(R)$. For a set of relaying nodes $R(s)$, the outage probability is given as:

$$
P_{\text {out }}[I<R]=\sum_{R(s)} \operatorname{Pr}[I<R \mid R(s)] \operatorname{Pr}[R(s)]
$$

In the sequel, we focus on high SNR analysis of the outage probability developed in [11], [12] for three scenarios depending on the spectrum acquisition capability of cognitive relay nodes in each cluster.

1) Perfect Spectrum Acquisition: This is the idealistic scenario where the relay nodes always acquire the spectrum holes successfully whenever they are available. Thus, this system is equivalent to the cooperative diversity scenario [11], [12] where relays have dedicated resources. Its performance is considered here to obtain an upper bound on the performance. In this case, the probability of relay node $r_{i}$ to be in the relaying set is

$$
\operatorname{Pr}\left[r_{i} \in R(s)\right]=\exp \left(-\lambda_{s r_{i}} \frac{2^{(M+1) R}-1}{S N R}\right)
$$

Since outage occurs independently for relay nodes, we have the following high SNR approximation

$$
\operatorname{Pr}[R(s)] \sim\left(\frac{2^{(M+1) R}-1}{S N R}\right)^{M-|R(s)|} \prod_{r_{i} \notin R(s)} \lambda_{s r_{i}}
$$

where $|R(s)|$ is the cardinality of the set, $R(s)$. The outage probability for RDF is given by [12]

$$
P_{\text {out }} \sim\left[\frac{2^{(M+1) R}-1}{S N R}\right]^{M+1} \Gamma_{P_{R D F}}
$$


where $\Gamma_{P_{R D F}}$ is given by

$$
\Gamma_{P_{R D F}}=\lambda_{s d} \sum_{R(s)} \prod_{r_{i} \in R(s)} \lambda_{r_{i} d} \prod_{r_{i} \notin R(s)} \lambda_{s r_{i}} \frac{1}{(|R(s)|+1) !}
$$

where $|R(s)| \in[0, M]$. In the sequel, we consider the asymmetric network where $\lambda_{s d} \neq \lambda_{s r_{i}} \neq \lambda_{s d_{i}}$. Given $M$, $\Gamma_{P_{R D F}}$ is given by a recursive formula for $M \geq 2$ :

$\Gamma_{P_{R D F_{M}}}(r)=\lambda_{s r_{M}} \Gamma_{P_{R D F_{M-1}}}(r)+\lambda_{r_{M} d} \Gamma_{P_{R D F_{M-1}}}(r+1)$

with $\Gamma_{P_{R D F_{1}}}(r)=\lambda_{s d} \lambda_{s r_{1}} /(r) !+\lambda_{s d} \lambda_{r_{1} d} /(r+1)$ ! [15].

Similarly, we get the outage probability of NDF as:

$$
P_{\text {out }} \sim\left[\frac{1}{S N R}\right]^{M+1}\left(2^{(M+1) R}-1\right)^{M} \Gamma_{P_{N D F}}
$$

where $\Gamma_{P_{N D F}}$ is given by

$$
\begin{aligned}
\Gamma_{P_{N D F}}= & \lambda_{s d} \sum_{R(s)}\left(2^{(M+1) R}-1\right)^{-|R(s)|} \prod_{r_{i} \in R(s)} \\
& \times \lambda_{r_{i} d} \prod_{r_{i} \notin R(s)} \lambda_{s r_{i}} G_{|R(s)|+1}((M+1) R)
\end{aligned}
$$

where $|R(s)| \in[0, M]$. Given $M, \Gamma_{P_{N D F}}$ is found by a treebased algorithm. We define $T(M, r)$ as follows.

$$
\begin{aligned}
T(M, r)= & \lambda_{s d} \lambda_{s r_{1}}\left(2^{(M+1) R}-1\right)^{-(r-1)} G_{r}((M+1) R) \\
& +\lambda_{s d} \lambda_{r_{1} d}\left(2^{(M+1) R}-1\right)^{-r} G_{r+1}((M+1) R)
\end{aligned}
$$

where $G_{k}(t)$ is defined as:

$$
G_{k}(t)=\int_{0}^{t} G_{k-1}(t-x) 2^{x} \ln (2) d x, \quad k=2,3, \ldots
$$

and $G_{1}(t)=2^{t}-1$. We construct the tree diagram by starting with $T(1,1)$, creating a left branch by increasing $M$ by one and a right branch by increasing $M$ and $r$ by one, respectively. Each branch is multiplied by a weight $L_{i}$ and $R_{i}, i \geq 2$. Then $\Gamma_{P_{N D F}}$ for certain $M$ is given by weighted sum of all nodes in the same level of the tree. $L_{i}$ and $R_{i}$ are given by $\lambda_{s r_{i}}$ and $\lambda_{r_{i} d}$, respectively.

Since there is no decoding constraint for AF, the outage probability for AF is given as [15]:

$$
P_{\text {out }} \sim\left[\frac{2^{(M+1) R}-1}{S N R}\right]^{M+1} \underbrace{\lambda_{s d} \prod_{i=1}^{M}\left(\lambda_{s r_{i}}+\lambda_{r_{i} d}\right) \frac{1}{(M+1) !}}_{\Gamma_{P_{A F}}}
$$

We note that $\Gamma_{P_{R D F}}, \Gamma_{P_{N D F}}$, and $\Gamma_{P_{A F}}$ are unchanged under permutations of $\lambda_{s r_{i}}$ and $\lambda_{r_{i} d}, i=1, \ldots, M$. We conclude that from the preceding analysis, that for each relaying scheme, we observe the full diversity order of $\mathrm{M}+1$.

2) Imperfect Spectrum Acquisition: A realistic scenario is that the potential relay nodes may not always be able to acquire the spectrum hole successfully, i.e., a spectrum opportunity is overlooked. Then for relaying techniques that involve decoding, i.e., RDF and NDF, the probability of relay node $r_{i}$ to be in the relaying set is

$$
\operatorname{Pr}\left[r_{i} \in R(s)\right]=\exp \left(-\lambda_{s r_{i}} \frac{2^{(M+1) R}-1}{S N R}\right) P_{d} .
$$

where we assume that each relay node acquires the spectrum hole with the same probability of $P_{d}$, and the corresponding false-alarm probability is $P_{f}$, as given in [6]. In this scenario, the outage probability is given by

$$
\begin{aligned}
P_{\text {out }}=\sum_{k=0}^{M} \sum_{R(s)} & \operatorname{Pr}[I<R \mid R(s), K=k] \\
& \times \operatorname{Pr}[R(s) \mid K=k] \operatorname{Pr}[K=k]
\end{aligned}
$$

where $K$ is the number of potential relay nodes that acquire spectrum holes successfully with $P_{d}$. Then, $\operatorname{Pr}[K=k]$ is

$$
\operatorname{Pr}[K=k]=\left(\begin{array}{c}
M \\
k
\end{array}\right) P_{d}^{k}\left(1-P_{d}\right)^{M-k}
$$

$\operatorname{Pr}[R(s) \mid K=k]$ as $S N R \rightarrow \infty$ is given by

$$
\left(\frac{2^{(M+1) R}-1}{S N R}\right)^{k-|R(s)|} \prod_{r_{i} \in R(s)} P_{d} \prod_{r_{i} \notin R(s)} \lambda_{s r_{i}}
$$

$\operatorname{Pr}\left[I_{R D F}<R \mid R(s), K=k\right]$ as $S N R \rightarrow \infty$ is given by

$$
\left[\frac{2^{(M+1) R}-1}{S N R}\right]^{|R(s)|+1} \times \lambda_{s d} \prod_{r_{i} \in R(s)} \lambda_{r_{i}} \frac{1}{(|R(s)|+1) !}
$$

Similarly, $\operatorname{Pr}\left[I_{N D F}<R \mid R(s), K=k\right]$ as $S N R \rightarrow \infty$ is given by

$$
\left[\frac{1}{S N R}\right]^{|R(s)|+1} \times \lambda_{s d} \prod_{r_{i} \in R(s)} \lambda_{r_{i} d} G_{|R(s)|+1}((M+1) R)
$$

Substituting (17), (18), and (19) into (16), the outage probability for RDF is given by

$P_{\text {out }} \sim \sum_{k=0}^{M}\left[\frac{2^{(M+1) R}-1}{S N R}\right]^{k+1}\left(\begin{array}{c}M \\ k\end{array}\right) P_{d}^{k}\left(1-P_{d}\right)^{M-k} \Gamma_{I_{R D F}}$

where $\Gamma_{I_{R D F}}$ is

$$
\lambda_{s d} \sum_{R(s)} \prod_{r_{i} \notin R(s)} \lambda_{s r_{i}} \prod_{r_{i} \in R(s)} P_{d} \prod_{r_{i} \in R(s)} \lambda_{r_{i}} d \frac{1}{(|R(s)|+1) !}
$$

with $|R(s)| \in[0, k]$. Given $k, \Gamma_{I_{R D F}}$ is found by a recursive formula $k \geq 2$ :

$$
\Gamma_{I_{R D F_{k}}}(r)=\lambda_{s r_{k}} \Gamma_{I_{R D F_{k-1}}}(r)+\lambda_{r_{k} d} P_{d} \Gamma_{I_{R D F_{k-1}}}(r+1)
$$

with $\Gamma_{P_{R D F_{0}}}(r)=\lambda_{s d}$ and $\Gamma_{I_{R D F_{1}}}(r)=\lambda_{s d} \lambda_{s r_{1}} /(r) !+$ $\lambda_{s d} \lambda_{r_{1} d} P_{d} /(r+1) !$.

Similarly, substituting (17), (18), and (20) into (16), the outage probability for NDF is given by

$$
\begin{aligned}
P_{\text {out }} \sim & \sum_{k=0}^{M}\left[\frac{1}{S N R}\right]^{k+1}\left(2^{(M+1) R}-1\right)^{k} \\
& \times\left(\begin{array}{c}
M \\
k
\end{array}\right) P_{d}^{k}\left(1-P_{d}\right)^{M-k} \Gamma_{I_{N D F}}
\end{aligned}
$$


where $\Gamma_{I_{N D F}}$ is given by

$$
\begin{aligned}
\lambda_{s d} & \sum_{R(s)}\left(2^{(M+1) R}-1\right)^{-|R(s)|} \prod_{r_{i} \notin R(s)} \lambda_{s r_{i}} \\
& \times \prod_{r_{i} \in R(s)} P_{d} \prod_{r_{i} \in R(s)} \lambda_{r_{i} d} G_{|R(s)|+1}((M+1) R)
\end{aligned}
$$

with $|R(s)| \in[0, k]$. Given $k, \Gamma_{I_{N D F}}$ is given by a recursive formula for $k \geq 2$ :

$$
\Gamma_{I_{N D F_{k}}}(r)=\lambda_{s r_{k}} \Gamma_{I_{N D F_{k-1}}}(r)+\lambda_{r_{k} d} P_{d} \Gamma_{I_{N D F_{k-1}}}(r+1)
$$

with the following initial values at $k=0$ and 1 , respectively.

$$
\begin{gathered}
\Gamma_{I_{N D F_{0}}}(r)=\lambda_{s d} G_{1}((M+1) R) \\
\Gamma_{I_{N D F_{1}}}(r)=\lambda_{s d} \lambda_{s r_{1}}\left(2^{(M+1) R}-1\right)^{-(r-1)} G_{r}((M+1) R) \\
+\lambda_{s d} \lambda_{r_{1} d} P_{d}\left(2^{(M+1) R}-1\right)^{-r} G_{r+1}((M+1) R) .
\end{gathered}
$$

Since for AF, there is no decodability constraints, the relay node only needs to correctly acquire the spectrum, and we have

$$
\operatorname{Pr}[R(s)]=\prod_{r_{i} \in R(s)} P_{d} \prod_{r_{i} \notin R(s)}\left(1-P_{d}\right)
$$

The outage probability for AF is given by

$$
\begin{aligned}
P_{\text {out }} \sim \sum_{R(s)} & {\left[\frac{2^{(M+1) R}-1}{S N R}\right]^{|R(s)|+1} \lambda_{s d} \prod_{r_{i} \in R(s)}\left(\lambda_{s r_{i}}+\lambda_{r_{i} d}\right) } \\
& \times \prod_{r_{i} \in R(s)} P_{d} \prod_{r_{i} \notin R(s)}\left(1-P_{d}\right) \frac{1}{(|R(s)|+1) !}
\end{aligned}
$$

where $P_{\text {out }}=\Gamma_{I_{A F}}$. Given $M, \Gamma_{I_{A F}}$ is again found by using tree algorithm as in (12). We define $T(M, r)$ as follows.

$$
\begin{aligned}
T(M, r)= & {\left[\frac{2^{(M+1) R}-1}{S N R}\right]^{r} \lambda_{s d}\left(1-P_{d}\right) /(r) ! } \\
& +\left[\frac{2^{(M+1) R}-1}{S N R}\right]^{r+1} \lambda_{s d}\left(\lambda_{s r_{1}}+\lambda_{r_{1} d}\right) P_{d} /(r+1) !
\end{aligned}
$$

$L_{i}$ and $R_{i}$ are given by $\left(1-P_{d}\right)$ and $\left(\lambda_{s r_{i}}+\lambda_{r_{i} d}\right) P_{d}, \forall i \geq$ 2 , respectively. We note that $\Gamma_{I_{R D F}}, \Gamma_{I_{N D F}}$, and $\Gamma_{I_{A F}}$ are unchanged under permutations of $\lambda_{s r_{i}}$ and $\lambda_{r_{i} d}, i=1, \ldots, M$. We note that the imperfect spectrum acquisition scenario does not achieve full diversity.

3) Imperfect Spectrum Acquisition with Intra-Cluster Cooperation: We can improve the performance of spectrum sensing if neighboring relay nodes of a potential relay node in each cluster can cooperate with the potential relay node by sharing the spectrum-sensing information. This is termed intra-cluster cooperation. For $N$ cooperating nodes, the potential relay node decides in favor of the presence of a spectrum hole if at least one of the cooperating nodes detects it. The probabilities of detection and false-alarm for intra-cluster cooperation are [16]

$$
C_{d}=1-\left(1-P_{d}\right)^{N+1}, \quad C_{f}=1-\left(1-P_{f}\right)^{N+1}
$$

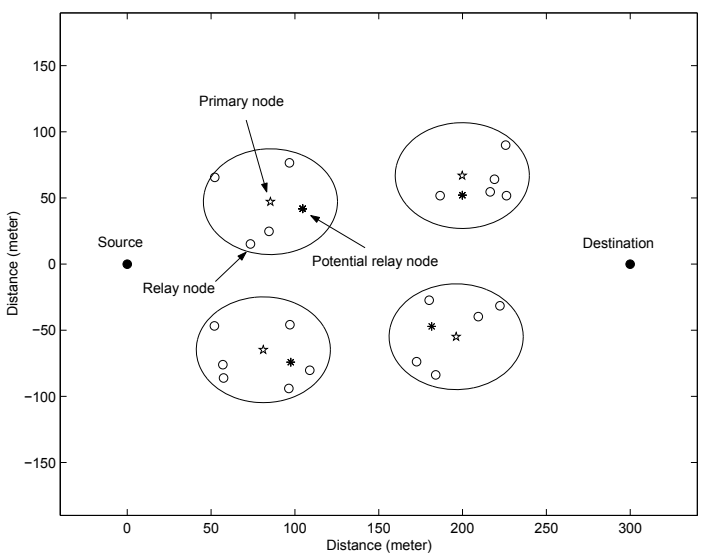

Fig. 2. Model of a spectrum-sensing opportunistic wireless relay network

We show that given $M$ and $C_{d}$, full diversity can be achieved if there are $N$ cooperating nodes, in each cluster satisfying the following:

$$
\Omega\left(M, S N R, C_{d}, N\right) \approx 1
$$

where $\Omega\left(M, S N R, C_{d}, N\right)$ for RDF and NDF is given by

$$
\frac{1}{\Gamma_{P}} \sum_{k=0}^{M}\left[\frac{2^{(M+1) R}-1}{S N R}\right]^{k-M}\left(\begin{array}{c}
M \\
k
\end{array}\right) C_{d}^{k}\left(1-C_{d}\right)^{M-k} \Gamma_{I}
$$

where $\Gamma_{I}$ and $\Gamma_{P}$ are equal to $\Gamma_{I_{R D F}}\left(\right.$ or $\left.\Gamma_{I_{N D F}}\right)$ and $\Gamma_{P_{R D F}}$ (or $\left.\Gamma_{P_{N D F}}\right)$, respectively.

For $\mathrm{AF}, \Omega\left(M, S N R, C_{d}, N\right)$ is given by

$$
\frac{1}{\Gamma_{P_{A F}}}\left[\frac{2^{(M+1) R}-1}{S N R}\right]^{-(M+1)} \Gamma_{I_{A F}}
$$

Equation (33) serves as a design rule: we can determine the number of cooperating nodes necessary for each scheme to have (34) and (35) sufficiently close to 1 and conclude that having at least that many cooperating nodes will achieve full diversity. It can easily be seen that when $C_{d}$ becomes close to one, (33) is satisfied and we can find the corresponding necessary number of cooperating nodes numerically.

\section{Numerical Results AND Discussion}

In this section, we present numerical results to support our analysis. We consider a network consisting of a source and a destination $300 \mathrm{~m}$ apart, and $M=4$ clusters that are distributed in a $300 \times 300 \mathrm{~m}^{2}$ square area, as shown in Figure 2. The radius of each cluster is $40 \mathrm{~m}$. Each cluster consists of a primary node placed in the center and group of relay nodes. Relay nodes that are closest to each primary user are chosen as potential relay nodes. We assume that we have $1 / \lambda_{s d}=C / d_{s d}^{\alpha}, 1 / \lambda_{s r_{i}}=C / d_{s r_{i}}^{\alpha}, 1 / \lambda_{r_{i} d}=C / d_{r_{i} d}^{\alpha}$, where $d_{A B}$ is the distance between node $A$ and $B$, and $\alpha$ is the path-loss exponent. $C=G_{t} G_{r} \lambda^{2} /(4 \pi)^{2} L$, where $G_{t}$ is the transmitter antenna gain, $G_{r}$ is the receiver antenna gain, $\lambda$ is the wavelength, and $L$ is the system loss factor not related to propagation $(L \geq 1)$. Throughout the numerical results, the values $\alpha=3, G_{t}=G_{r}=1, \lambda=1 / 3 m$ (carrier frequency $f=900 \mathrm{MHz}$ ), $L=1$, are used. The AWGN variances on all communication links are assumed to be $10^{-10}$. The 


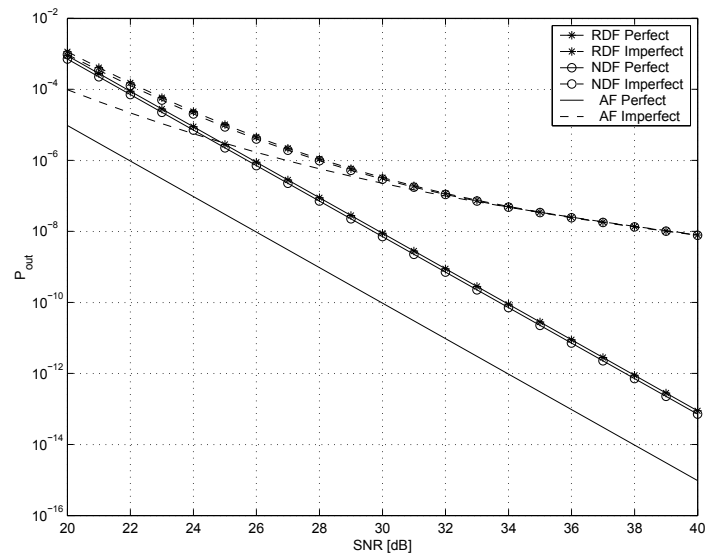

Fig. 3. Outage nrobahilities at high SNR region

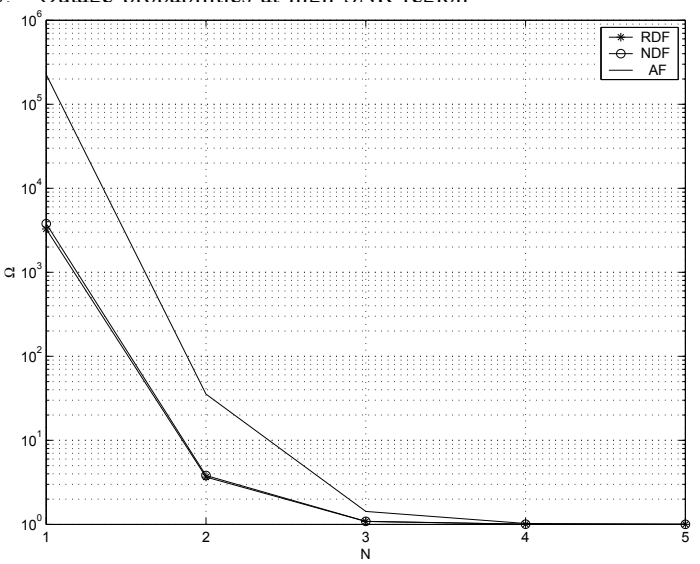

Fig. 4. The number of required cooperating nodes for achieving full diversity

probability of detection, $P_{d}$, is the one that is minimum of $P_{d_{i}}, i=1, \ldots, 4$, which is the probability of detection at $i$ th potential relay node. Values of other parameters are: $R=1$, $P_{f}=10^{-2}$, and $C_{f}=10^{-2}$.

Figure 3 shows the outage probabilities corresponding to the three relay transmission schemes, AF, NDF, and RDF. We observe that all three relay transmission schemes for the perfect spectrum acquisition achieve full-diversity. However, full-diversity is not achieved for the imperfect spectrum acquisition. We observe that AF performs best and NDF performs better than RDF.

Figure 4 plots $\Omega\left(M, S N R, C_{d}, N\right)$ for all three schemes to find the required number of cooperating node in each cluster for achieving full-diversity. We observe that $N=3$ is necessary to achieve full-diversity for NDF and RDF. $N=4$ is the required number of cooperating nodes to achieve full-diversity for AF. Thus, our conclusion is that for achieving full-diversity, we need (i) intra-cluster cooperation in spectrum sensing with a relatively modest number of cognitive relays, and (ii) intercluster cooperation for relaying the source information to the destination.

\section{CONCLUSION}

We have analyzed the outage performance of spectrumsensing opportunistic wireless relay networks where a single source-destination pair is assisted by a group of cognitive relay nodes. We have considered the asymmetric network scenario and three relay transmission schemes (RDF, NDF, and $\mathrm{AF}$ ). We have analyzed the high SNR approximation of outage probability in order to study the diversity order. Regardless of the relay transmission scheme used, we observe that when the potential relay nodes have spectrum acquisition errors, full diversity may not always be achieved. To overcome this performance deficiency, we have investigated intra-cluster cooperation, which allows the neighboring relay nodes in a cluster to collaborate with a potential relay node in acquiring the spectrum hole. The intra-cluster cooperation is shown to improve the outage performance. Specifically, we observe that the full diversity is achieved if the proper number of neighboring relay nodes in each cluster participate in intracluster cooperation.

\section{REFERENCES}

[1] R. W. Broderson, A. Wolisz, D. Cabric, S. M. Mishra, and D. Willkomm. CORVUS : A cognitive radio approach for usage of virtual unlisenced spectrum. http://bwrc.eecs.berkeley.edu/Research/MCMA/CRWhitepaperfinal1.pdf.

[2] J. Mitola and G. Q. Maguire Jr. Cognitive radio: making software radios more personal. IEEE Personal Communications, 6(4):13 - 18, August 1999.

[3] S. Haykin. Cognitive radio: Brain-empowered wireless communications. IEEE Journal on Selected Areas in Communications, 23(2):201 - 220, February 2005

[4] A. Sahai, N. Hoven, and R. Tandra. Some fundamental limits on cognitive radio. In Allerton conference on Coomunications, Control, and Computing, 2004

[5] V. I. Kostylev. Energy detection of a signal with random amplitude. In IEEE International Conference on Communications, ICC'03, pages 1606 - 1610, May 2002.

[6] F. F. Digham, M. S. Alouini, and M. K. Simon. On the energy detection of unknown signals over fading channels. In IEEE International Conference on Communications, ICC'03, pages 3575 - 3579, May 2003.

[7] A. Ghasemi and E. S. Sousa. Collaborative spectrum sensing for opportunistic access in fading environments. In First IEEE International Symposium on New Frontiers in Dynamic Spectrum Access Networks, DySPAN'05, pages 131 - 136, November 2005.

[8] G. Ganesan and Y. Li. Agility improvement through cooperative diversity in cognitive radio. In IEEE Global Telecommunications Conference, GLOBECOM' 05, pages 2505 - 2509, 28 November - 2 December 2005.

[9] A. Sendonaris, E. Erkip, and B. Aazhang. User cooperation diversity part I: System description; part II: Implementation, aspects and performance analysis. IEEE Transactions on Communications, 51(11):1927 - 1948, November 2003.

[10] A. Høst-Madsen and J. Zhang. Capacity bounds and power allocation in wireless relay channel. IEEE Transactions on Information Theory, 51(6):2020 - 2040, June 2005.

[11] J. N. Laneman, D. N. C. Tse, and G. W. Wornell. Cooperative diversity in wireless networks: Efficient protocols and outage behavior. IEEE Transactions on Information Theory, 50(12):3062 - 3080, December 2004.

[12] J. N. Laneman and G. W. Wornell. Distributed space-time-coded protocols for exploiting cooperative diversity in wireless networks. IEEE Transactions on Information Theory, 49(10):2415 - 2425, October 2003.

[13] K. Lee and A. Yener. Outage performance of cognitive wireless relay networks. In IEEE Global Telecommunications Conference, GLOBECOM'06, 27 November-1 December 2006.

[14] S. Mangold, A. Jarosch, and C. Monney. Operator assisted cognitive radio and dynamic spectrum assignment with dual beacons - detailed evaluation. In First International Conference on Communication System Software and Middleware, Comsware'06, pages 1-6, January 2006.

[15] J. N. Laneman. Network coding gain of cooperative diversity. In IEEE Military Communications Conference, MILCOM'04, pages $106-112$, November 2004.

[16] P. K. Varshney. Distributed Detection and Data Fusion. Springer, 1997. 